\title{
Pratiques
}

Linguistique, littérature, didactique

$159-160 \mid 2013$

Le figement en débat

\section{Figement et défigement dans la traduction}

L'exemple d'un roman de Günter Grass

\section{Heinz-Helmut Lüger}

\section{OpenEdition}

Journals

Édition électronique

URL : http://journals.openedition.org/pratiques/2892

DOI : $10.4000 /$ pratiques.2892

ISSN : 2425-2042

Éditeur

Centre de recherche sur les médiations (CREM)

\section{Édition imprimée}

Date de publication : 15 décembre 2013

Pagination : 165-178

\section{Référence électronique}

Heinz-Helmut Lüger, «Figement et défigement dans la traduction », Pratiques [En ligne], 159-160 |

2013, mis en ligne le 30 juin 2016, consulté le 14 novembre 2019. URL : http://

journals.openedition.org/pratiques/2892 ; DOI : 10.4000/pratiques.2892

(c) Tous droits réservés 


\title{
Figement et défigement dans la traduction. L'exemple d'un roman de Günter Grass
}

\author{
Heinz-Helmut Lüger
}

Universität Koblenz-Landau

Dans quelle mesure les expressions toutes faites peuvent-elles poser problème pour la traduction? Quelle serait la différence par rapport aux constructions «libres » et non figées ? Faut-il accorder une attention particulière aux expressions idiomatiques et à leur emploi créatif ? Le but de l'étude qui suit sera donc de montrer pourquoi la recherche d'équivalence (sémantique et pragmatique) dans une langue cible n'est pas toujours facile et comment, en ce qui concerne les expressions figées, la traduisibilité peut être parfois limitée. Les exemples cités dans cet article sont tirés d'un texte littéraire unanimement considéré comme intéressant et complexe : Günter Grass, Ein weites Feld ${ }^{(1)}$.

\section{Expressions figées, emploi créatif et texte littéraire}

Günter Grass fait partie de ces auteurs qui se distinguent par un style allusif, original et exigeant. Très souvent, le lecteur se voit confronté à une situation où le choix entre plusieurs interprétations paraît assez difficile. Il y a également des allusions historiques, des citations plus ou moins identifiables, des mots composés surprenants ou des métaphores énigmatiques, des traits caractéristiques qui demandent donc un effort considérable au lecteur. L'attribution du sens représente en effet un travail intensif qui ressemble parfois à une aventure et qui n'a pas de solution simple. Le roman Ein weites Feld, paru en 1995, correspond, pour des raisons diverses, à ce type de texte ${ }^{(2)}$.

En ce qui concerne la réalisation stylistique de ce texte les expressions figées

(1) Je tiens à remercier ma collègue Françoise Hammer qui a généreusement accepté la relecture du texte français.

(2) Les citations se réfèrent à l'édition suivante: Grass, G. (1995): Ein weites Feld. Roman. Göttingen, Steidl. Comme base de comparaison seront consultées deux traductions : Toute une histoire. Roman. Traduit de l'allemand par Claude Porcell et Bernard Lortholary. Paris, Seuil 1997 ; È una lunga storia. Traduzione di Claudio Groff. Torino, Einaudi 1998. Dans les extraits seront indiqués le chapitre et la page. 
(ou unités phraséologiques) jouent un rôle important. Seront désignées ainsi des expressions polylexicales qui se composent au moins de deux éléments autosémantiques. Dans notre texte de référence on trouve une multitude de constructions de ce genre ${ }^{(3)}$ :

- syntagmes verbaux

eine Kröte schlucken $\rightarrow \quad$ avaler la couleuvre/ inghiottire (ingoiare) il rospo

- syntagmes nominaux

die gute Sache $\rightarrow$ la bonne cause / il fatto positivo

- syntagmes adverbiaux

partout nicht $\rightarrow$ à aucun prix / non assolutamente

- syntagmes adjectivaux

arm an Worten $\rightarrow$ avare de mots / avaro di parole

- syntagmes prépositionnels

unter Palmen $\rightarrow$ sous les cocotiers/sotto le palme

Évidemment, on pourrait encore approfondir cette subdivision globale, mais comme le but de cet article n' est pas de fournir une nouvelle classification des unités phraséologiques, un renvoi à d'autres travaux paraît suffisant ${ }^{(4)}$. Les exemples servent à montrer comment certains traits spécifiques peuvent varier : le degré de fixité n'est pas le même pour les syntagmes arm an Worten et gute Sache ou 1'expression eine Kröte schlucken qui se caractérise, comparée aux autres exemples, par une opacité sémantique plus forte. De plus, le figement ne se limite pas au niveau des syntagmes, mais peut concerner également des phrases entières; c'est le cas par exemple des proverbes, lieux communs, slogans et « citations proverbialisées » ou «citations stéréotypées » ${ }^{(5)}$. Cependant, on peut constater que l'emploi des proverbes et des lieux communs, au moins dans leur forme originale, est plutôt rare dans notre texte de référence. On trouve tout au plus des phrases figées (1) ${ }^{(6)}$, des slogans (2) ou des citations littéraires plus ou moins connues (3):

(1) Da ist die Luft raus! (III, 54) - (le cœur n'y est plus! (52))

(2) Dabeisein ist alles! (III, 63) - (L'essentiel est de participer! (60))

(3) „Sage nichts Französisches. Das verdrießt mich immer.“(VI, 118) -

(«Rien de français, je t'en prie. Cela me fâche toujours. » $(110))^{(7)}$

Le nombre limité de phrasèmes propositionnels devrait s'expliquer par le fait

(3) Tous les exemples proviennent du $19^{\mathrm{e}}$ chapitre du roman.

(4) $C f$. Fleischer (1997: 110-161), Burger (1998:33-55), Lüger (1999: 27-53), González Rey (2002: 41-63).

(5) Ce terme a été proposé par Schapira (1999: 142), il correspond à ce qu'on désigne en allemand par «geflügeltes Wort», donc des citations qui sont devenues des formules usuelles ; pour plus de détails cf. Burger et al. (1982: 43-56). En ce qui conerne le "préformé » dans le langage, on a tendance à élargir le champ de la phraséologie et à prendre aussi en considération aussi le plan textuel (Stein 2001, Schmale 2011).

(6) L'expression phrases figées reprend le terme festgeprägte Sätze de Reichstein (1973); il désigne des unités phraséologiques à valeur de phrase qui ne sont pas totalement figées et contiennent un élément qui les relie au contexte (ex. : Da liegt der Hund begraben).

(7) La source de cette citation est, comme souvent chez Grass, le roman Der Stechlin de Theodor Fontane. 
que ces unités représentent un moyen linguistique très marqué et relativement lourd qui s'intègre mal dans un style original et chargé d'allusions intertextuelles. Plus typiques sont par contre des schémas syntaxiques figés qui ressemblent à des constructions préformées et dont le plan lexical n'est pourtant pas fixe :

(4) Nichts steht für immer. (I, 17) - (Rien n'est éternel. (19))

(5) Niemand siegt ungestraft. (XX, 411) - (Il n'y a pas de victoire impunie. (379))

Il s'agit donc, dans la plupart des cas, des formes d'expression qui correspondent avec leurs marques linguistiques aux lieux communs ou proverbes, mais qui ne font pas partie du stock des constructions lexicalisées. Ces schémas syntaxiques permettent, grâce à leur caractère générique et concis, de suggérer des vérités éternelles ou universelles. Dans leur contexte ils sont souvent utilisés comme arguments justifiant des commentaires sur les actualités politiques, notamment sur la chute du mur de Berlin et sur l'histoire de la Prusse ou de l'Allemagne ; l'énoncé (4) peut en effet être compris comme une remarque ironique, (5) transmet un principe général qui soutient une mise en garde concernant l'avenir politique tout en tenant compte du passé historique avant la réunification allemande.

Quant aux citations, proverbialisées ou non, elles servent souvent comme point de départ pour attirer l'attention sur une question importante. En même temps, elles peuvent donner accès à des perspectives différentes ou à des contextes multiples. Dans l'extrait suivant les expressions soulignées sont susceptibles d'évoquer plusieurs scénarios :

(6) « [...] Was ich im verflossenen November auf dem Alex gesagt habe, als da Hunderttausende standen, das gilt immer noch: «Eine neue Zeit bricht an! Ich glaube, eine bessere und glücklichere! Und wenn nicht eine glücklichere, so doch mindestens eine Zeit mit mehr Sauerstoff in der Luft, eine Zeit, in der wir besser atmen könen. Und je freier man atmet, je mehr lebt man!) » (VII, 137-138)

Tout d'abord, il s'agit d'un dialogue entre les deux protagonistes du roman où les récents changements en Allemagne sont discutés d'une manière assez controversée. Ensuite la citation qui est insérée dans un énoncé plus long, nous mène directement à un texte de Theodor Fontane, plus précisément : au $29^{\mathrm{e}}$ chapitre du Stechlin, le roman déjà cité plus haut. Ici, ce sont les réflexions critiques et les idées libérales du pasteur Lorenzen qui invitent le lecteur à se plonger dans 1'histoire prussienne du $19^{\mathrm{e}}$ siècle (6').

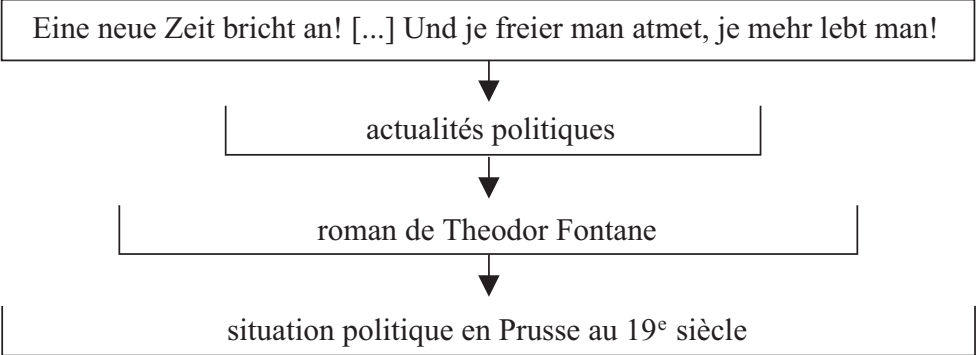

En ce qui concerne les phraséologismes à valeur de syntagme, p. ex. des expressions idiomatiques, on peut constater deux tendances : un emploi des formes lexi- 
calisées et un emploi des formes modifiées. Les procédés apportant de telles variations formelles peuvent être multiples :

- substitution

(den Teufel an die Wand malen $\rightarrow$ den Teufel an die Wand schwatzen (VII, 137))

- expansion

(verkrachte Existenz $\rightarrow$ durch und durch verkrachte Existenz (VI, 127), in die Falle gehen / tappen $\rightarrow$ in die Ehefalle tappen (XXII, 432))

- réduction

(kein Sterbenswörtchen sagen $\rightarrow$ kein Wörtchen sagen (XXII, 446))

- contamination (+ expansion)

(ein Klotz am Bein sein + jmdm Knüppel zwischen die Beine werfen $\rightarrow$ mit ministeriellem Knüppel am Bein (VI, 126))

- dérivation déphraséologique

(mit jmdm Schritt halten $\rightarrow$ das schritthaltende Mädchen (XXII, 432))

Tous les exemples illustrent une mise en relief des unités en question et par là, une certaine oscillation entre la signification phraséologique et la signification littérale. En plus, ces variations soulignent la volonté de se servir des expressions toutes faites d'une manière créative et ludique pour produire des effets stylistiques suggestifs, surprenants et parfois amusants et mettre à l'épreuve les attentes linguistiques des lecteurs. S'y ajoutent des occurrences où c'est avant tout le contexte qui apporte des modifications. Ainsi, dans (7), l'emploi de la locution kein Blatt vor den Mund nehmen « parler franchement » n'a, à première vue, rien d'extraordinaire :

(7) Wenngleich er Menzel höher schätzte, war Liebermann ganz nach seinem Geschmack. Der nahm kein Blatt vor den Mund. (III, 51) ${ }^{(8)}$

La personne en question est caractérisée comme quelqu'un qui ne craint pas de dire la vérité et de s'exprimer sans ménagement — comme la signification phraséologique de kein Blatt vor den Mund nehmen le suggère. Mais quand on regarde le contexte de l'énoncé on peut constater que quelques lignes plus bas le lexème Blatt est repris deux fois ( "Liebermann zeichnete Blatt nach Blatt, war aber nicht stumm dabei » (52)); en plus, les syntagmes verbaux nicht stumm sein et die Tage nur noch mit Schimpfen verbringen (52) confirment le lien avec l'activité communicative indiquée par l'expression idiomatique. En somme, ces éléments contextuels visent à attirer l'attention sur les constituants, notamment sur Blatt et sa signification littérale et contribuent à ré-activer le sens compositionnel de kein Blatt vor den Mund nehmen (Fig. 1).

(8) Selon Röhrich (1982 : 133), l'expression kein Blatt vor den Mund nehmen peut être ramenée à une ancienne mesure des acteurs au théâtre qui cachaient leur visage derrière des feuilles afin de pouvoir se prononcer librement sans être rendu responsables : «Die Redensart spiegelt eine alte Theatersitte wider. Die Schauspieler machten sich unkenntlich, indem sie Blätter vor ihr Gesicht hielten. Sie konnten dann manches vorbringen, ohne später dafür zur Rechenschaft gezogen zu werden. [...] Wer also kein Blatt vor den Mund nimmt, will sich nicht verstecken, sondern offen seine Meinung bekennen. " Sous cet angle, l'expression française ne pas mâcher ses mots ne serait donc pas l'équivalent total. 


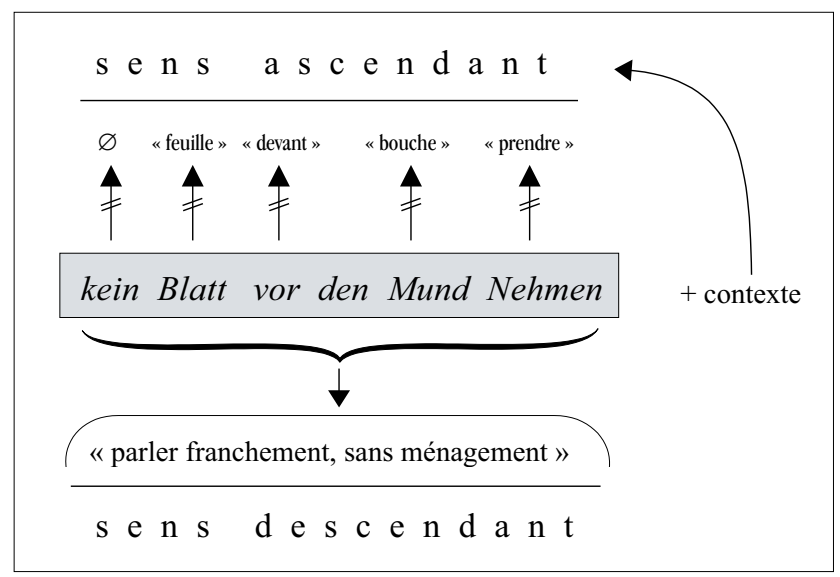

Fig. 1 : Sens global/descendant et remotivation

Même si la remotivation de l'unité phraséologique n'est probablement pas perçue par tous les lecteurs, il s'agit d'un procédé très fréquent dans l'œuvre de Günter Grass ${ }^{(9)}$. Il repose sur un emploi insolite entraînant une certaine rupture de la routine interprétative, un effet qui est bien connu dans la phraséologie et déjà décrit à plusieurs reprises. Deux phénomènes sont d'une importance centrale ici : Plus un groupement de mots est figé, plus la signification des composants est globalisée, neutralisée, opacifiée ; par conséquent, il y a, en cas d'emploi textuel, une « rupture entre le sens littéral et le sens idiomatique» (Gréciano 1983 : 175). Ou, en partant des termes « sens ascendant » et « sens descendant» :

«Selon le degré de figement de l'expression, son sens descendant peut être plus ou moins établi et dominant, et corrélativement son sens ascendant plus ou moins libre et accessible. Plus une expression est figée, plus son sens codé descendant est dominant, et moins son sens ascendant est libre et accessible. À terme, lorsque la lexicalisation d'une expression s'achève, son sens ascendant finit par disparâ̂tre. » (Perrin $2011: 84)$

Le deuxième phénomène résulte du fait que le figement des groupes de mots n'atteint que rarement un degré absolu. D'abord, un grand nombre de locutions reste partiellement motivées ; p. ex. dans sich um Kopf und Kragen reden ou verkrachte Existenz les composants reden et Existenz gardent leur signification littérale. Et puis, il y a des modifications sur le plan formel et des mesures contextuelles qui peuvent, comme les exemples l'ont démontré, favoriser une lecture littérale sans exclure toutefois une compréhension globale ou phraséologique.

L'emploi créatif des groupes de mots figés demande aux lecteurs une certaine disposition à se laisser inspirer par les jeux de mots, à accepter la coprésence de lectures différentes ou même opposées et à trouver un sens aux allusions et aux modifications des expressions respectives :

«Le lecteur reproduit l'actualisation d'une dimension sémantique inattendue, sinon l'intention ludique échoue. L'accomplissement de l'intention communicative dans la réaction du partenaire est donc une condition nécessaire. » (Gréciano $1983: 255)$

(9) Cf. Schweizer (1978), Koller (1992), Benger (2004), Lüger (2004). 
La densité sémantique et le style allusif de Grass ne facilitent pas toujours la réception, une constatation qui vaut surtout pour les lecteurs non germanophones. Un problème peut aussi se poser pour la traduction : Dans quelle mesure l'emploi ludique des expressions figées par exemple est-il vraiment traduisible dans une autre langue ? Cette question sera abordée dans le chapitre suivant à l'aide des traductions française et italienne du roman Ein weites Feld.

\section{Polyfonctionnalité et traduisibilité}

Plusieurs études ont déjà illustré d'une manière détaillée, et cela à partir de langues diverses et d'auteurs différents, qu'il est pratiquement impossible d'avoir des traductions qui correspondent cent pour cent au texte de départ; et même une équivalence fonctionnelle est difficile à atteindre ${ }^{(10)}$. Dans l'ensemble, on peut distinguer plusieurs cas de figure qui représentent plutôt des gradations sur une échelle continue entre des pôles 〈équivalence totale〉 et d'〈équivalence zéro〉. Les exemples et les commentaires qui suivent vont se concentrer sur quatre aspects : Le traducteur peut-il recourir à une construction figée analogue dans la langue cible (2.1) ? Y a-t-il, en cas de réponse négative, un groupe de mots préformé dont la signification peut être considérée comme équivalente (2.2.) ? Et quand une telle expression n'est pas disponible : À quel écart par rapport à l'énoncé de départ faut-il s'attendre (2.3) ? Ya-t-il des exemples où la traduction s'éloigne du texte original de telle façon qu'on ne peut parler que d'une équivalence zéro (2.4.) ?

2.1. Les cas, où il existe des formes analogues dans la langue de départ et dans une langue cible, ne sont pas très nombreux. Dans l'exemple (8), l'expression etw. an den Haaren herbeiziehen ne pose pas problème pour la traduction ${ }^{(11)}$ :

(8a) Zwar nörgelte sie, fand alles zu weitschweifig und - wie sie sagte - « an den Haaren herbeigezogen $»[\ldots]$. (XII, 249-250)

(8b) Certes elle pestait, trouvait tout cela trop circonstancié et, comme elle disait, « $\underline{\text { tiré }}$ par les cheveux » [...]. (231)

(8c) Certo brontolò, trovò che tutto saltava troppo di palo in frasca e - come disse « era tirato per i capelli $»[\ldots]$. (203)

Tandis que la traduction des expressions idiomatiques s'avère souvent problématique à cause de leur valeur évaluative, le traitement des schémas syntaxiques figés comme p. ex. Niemand siegt ungestraft (déjà cité en (5)) paraît moins difficile ; étant donné que ces constructions ne sont pas répertoriées comme des unités lexicalisées, une traduction mot à mot semble envisageable ici. Pour 1'expression citée on trouvera donc: Il n'y a pas de victoire impunie (XX, 379) et Nessuno vince impunemente (334). D'autres exemples confirment cette oberservation, même s'il s'agit parfois de contenus inhabituels.

(9) Aber was richtig ist, muß nicht wahr sein. (VII, 140) $\rightarrow$ Mais ce qui est exact n'est pas forcément vrai. (132) $\rightarrow$ Ma ciò che è esatto non deve per forza essere vero. (112)

(10) Koller (1974), (1994), Klimaszewska (1992), Ettinger (1994), Mohr-Elfadl (1999), Benger (2004), Dobrovol'skij (2004), Forgács (2004).

(11) Dans l'extrait italien on trouve même une locution métaphorique supplémentaire : saltare di palo in frasca (sauter du coq à l'âne) ce qui ne correspond que vaguement à weitschweifig («prolixe»). 
(10) Bruch ist besser als Ganzes! (I, 14) $\rightarrow$ Les parties valent mieux que le tout! (16) $\rightarrow$ Il frammento è meglio dell'insieme! (10)

Les choses ont tendance à se compliquer quand l'auteur commence à s'éloigner des formes établies : «L'interaction entre unité phraséologique et cotexte est plus riche, plus complexe dès qu'il y a modification. » (Mohr-Elfadl 1999: 403) Un des procédés préférés est l'adjonction d'un ou de plusieurs éléments ; dans (11) il y a une expansion à gauche du substantif Wort qui fait partie du phraséolexème $j m d n$ beim Wort nehmen :

(11a) Deshalb nahmen wir ihn nicht nur beim geplauderten und über Seiten und Verskolonnen hinweg zitatseligen Wort, sondern [...]. (III, 48)

(11b) Voilà pourquoi non seulement nous le prenions au mot, aux mots qu'il maniait en causeur et qu'il émaillait à plaisir de citations de pages et de poèmes entiers, mais [...]. (47)

(11c) Pertanto, quando si facevano quattro chiacchiere, noi non soltanto gli credevamo sulla parola felicemente citata al di là delle pagine e delle colonne di versi, ma [...]. (37)

La formulation est déjà assez complexe dans sa version allemande, et ce n'est pas le phraséolexème qui complique ici la tâche des traducteurs. On constate d'abord qu'il est pratiquement impossible de trouver une expression adéquate pour le mot composé zitatselig, un mot qui ne figure pas dans le lexique allemand et qui est une création de l'auteur. En (11b), l'unité jmdn beim Wort nehmen est mise en relief et puis cette longue adjonction de l'énoncé de départ se trouve paraphrasée et transformée en expansion à droite. Il se peut que cette version soit plus claire et plus compréhensible, mais rend-elle aussi l'originalité stylistique du texte source ? La traduction dans (11c) s'éloigne encore davantage du modèle allemand: On renforce la locution prendere qcn. in parola en soulignant la crédibilité avec credere a qcn. sulla parola, et, placée en tête de la phrase, la locution fare quattro chiacchiere con qcn. " faire une petite causette » met l'accent sur l'ambiance qui détermine la situation. Ici encore, tout est fait pour faciliter la compréhensibilité, mais une question subsiste : Ces moyens stylistiques sont-ils appropriés pour transmettre également le ton distancié voire sarcastique dont Grass se sert pour critiquer certaines methodes de mise en scène du monde littéraire ?

2.2. Dans certains cas, le traducteur a recours à des expressions figées dans la langue cible qui peuvent être considérées comme des synonymes ou quasi-synonymes. Mais souvent une différence sémantique semble inévitable. La locution aus dem Nähkästchen plaudern p. ex. ne connaît pas de forme analogue en français ou en italien. Les solutions proposées ne correspondent donc que partiellement:

(12) $[\ldots]$ insgeheim hoffend, er werde aus der Rolle fallen oder ein wenig aus dem Nähkästchen plaudern $(V, 85) \rightarrow$ en espérant secrètement qu'il sortirait du rôle ou que nous lui tirerions un peu les vers du nez $(80) \rightarrow$ sperando in segreto che facesse una magra figura o che spiattellasse qualcosa (66)

Contrairement à la locution allemande présupposant un acteur qui livre des informations de son plein gré, tirer les vers du nez à qn fait penser à une situation où une personne est traitée d'une certaine manière pour lui arracher des secrets. Dans la version italienne, le traducteur a préféré le verbe spiattellare qui de plus ne rend pas l'idée qu'il s'agit d'informations confidentielles ou secrètes. Pour la même raison fare pettegolezzi « bavarder » ne s'impose pas ici. 
Dans l'exemple suivant, il s'agit d'une phrase figée (es geht um die Wurst «c'est le moment décisif») qui est, grâce aux rapports métonymiques, étroitement liée à son entourage textuel : Wurst-scheibchenweise-überfuttern. Ainsi, une double lecture littérale et phraséologique est en effet possible. En (13b), la traduction française intègre la locution gagner/défendre son bifteck et déploie une isotopie sémantique correspondante : bifteck-petits morceaux - indigestion. Certes, on peut se demander si les deux unités phraséologiques sont vraiment comparables. De toute façon, l'image dans les deux expressions n'est pas la même et le fait qu'un moment décisif se présente n'est pas évoqué d'une manière identique ${ }^{(12)}$.

(13a) « War doch schon immer so, wenn's um die Wurst ging. Der Rest fürs Volk, und zwar scheibchenweise, damit sich ja keiner überfuttert... (VIII, 161)

(13b) «Ça a toujours été comme ça, dès qu'il est question du bifteck. Le peuple a les restes, et en tout petits morceaux, pour lui éviter l'indigestion... » (150)

(13c) «È sempre stato cosí, nei momenti decisivi. Il resto per il popolo, e a fettine sottili, in modo che nessuno si rimpinzi...» (128)

En (13c), le traducteur a choisi une autre stratégie : La phrase figée est rendue par une paraphrase non-phraséologique qui transmet très bien le message central. Mais les expressions fettine sottili et nessuno si rimpinzi n'entretiennent aucune liaison avec le contexte précédent, et la double lecture qui caractérise les versions allemande et française n'est plus possible ici. Déjà l'énoncé « il resto per il popolo » qui n'a pas de point de repère précis, paraît mal placé.

Comme l'auteur a une préférence marquée pour l'emploi créatif des expressions figées il n'est pas toujours possible de trouver une traduction précise. Cela est d'autant plus difficile qu'une locution peut aussi être combinée avec une figure rhétorique. La synchyse en (14) semble complètement intraduisible, y compris l'alternance entre sens global et littéral ; l'usage des expressions tomber mal et mancare la presa n'y change rien :

(14) was sie sagt, hat Hand und Fuß, selbst wenn es daneben greift oder fehltritt (XII, $252) \rightarrow$ ce qu'elle dit se tient, même quand ça tombe mal ou à côté (234) $\rightarrow$ quello che dice ha sempre un senso, anche quando manca la presa o sbaglia (205)

Un problème semblable se pose dans l'énoncé suivant, où une structure zeugmatique se trouve à la base de la version allemande :

(15) Ernahm Haltung an und den Hut ab (II, 34) $\rightarrow$ Droit comme un i, il ôta son chapeau

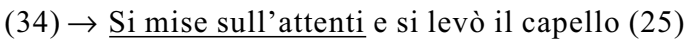

Dans les deux cas, Haltung annehmen est traduit par un phraséologisme dont l'expression mettersi sull'attenti correspond bien et reprend même l'aspect militaire demandé par le contexte, tandis que droit comme un i ne représente qu'une solution approximative. Par contre, les traductions font complètement abstraction du zeugme sémantique de l'énoncé source.

2.3. Très nombreux sont les exemples où il n'est pas possible de recourir à une expression figée et où la traduction doit se contenter d'une paraphrase plus ou moins appropriée.

(12) Benger (2004: 162) fait remarquer qu'il y aurait, en outre, une différence culturelle considérable entre les termes bifteck et Wurst; le dernier serait considéré comme une sorte de symbole culinaire d'identité nationale pour les Allemands... 
Comme il n'y a pas de véritable équivalent pour la phrase figée die Spatzen pfeifen es von den Dächern (16), on trouve deux traductions assez différentes : Le texte français renonce à cette expression imagée et propose après, en compensation, la tournure graisser la patte à qn, sans pour autant tenir compte du mot composé $t a-$ lerschwer et sans respecter le niveau du registre stylistique. La version italienne se rapproche davantage de l'original en choisissant le même type de phraséologisme (lo sanno anche $i$ sassil polli).

(16) Dabei pfiffen die Spatzen von den Dächern, wer an wen talerschwer Bestechungsgelder gezahlt hat. $(\mathrm{V}, 89) \rightarrow$ Avec ça, tout le monde vous racontait qui avait graissé la patte à qui. (83) $\rightarrow$ E poi lo sapevano anche i sassi, chi aveva cercato di corrompere chi con una montagna di soldi. (69)

Parfois, il est intéressant de voir comment une seule et même unité phraséologique a été transposée dans la langue cible. Prenons comme exemple la construction prédicative figée der Groschen fällt bei jmdm «il a enfin compris, il a pigé » ${ }^{(13)}$ :

(17) (a)

Fällt der Groschen? (I, 16)

Fällt der Groschen? (II, 41)

Fällt der Groschen? (XVIII, 376)

Will der Groschen nicht fallen?

(XX, 412) (b)

Vous pigez? (18)

Vous y êtes? (41)

Vous y êtes? (346)

Ça ne vient pas?

(380) (c)

Le viene qualche idea? (11)

Si accende la lampadina? (31)

Ci stiamo arrivando? (305)

Non si accende la lampadina?

(335)

Ce n'est pas par hasard que cette construction figée est utilisée à plusieurs reprises. Dès le premier chapitre, l'auteur s'efforce d'établir un rapport entre Groschen, fallen/ Fall et Mauerfall. Et en ce qui concerne la chute du mur, on trouve également des expressions comme groschengroße Mauerbröcklein, groschengroße Absprengsel ou Hinfälligkeit des Bauwerks ${ }^{(14)}$. Si on compare les passages respectifs aux versions française et italienne, on constate que ces rapports, quelle qu'en soit la raison, n'ont pas été repris. Et même pour l'unité phraséologique der Groschen fällt on trouve des traductions différentes ce qui rend déjà impossible cette parallélisation qui caractérise le texte source.

Comme plusieurs exemples l'ont déjà montré, les traductions mot à mot et les paraphrases n'aboutissent pas toujours à des solutions satisfaisantes, très souvent il faut s'attendre à des pertes ou à des décalages sémantiques et pragmatiques considérables. L'extrait suivant servira à illustrer encore plus concrètement une telle réduction.

(18a) [...] während Fontys Vater zwei Jahre nach dem Mauerbau davonging, um sich, nach gärtnerischer Redeweise, « die Radieschen von unten anzusehen» (XII, 254)

L'action verbale essentielle réalisée par cet énoncé consiste à informer le destinataire d'un événement qui a eu lieu dans le passé. Mais cet état de choses n'est pas présenté de manière neutre : Déjà le verbe davongehen transmet un aspect étrange, de toute façon, le lexème choisi ne correspond pas aux attentes linguistiques ici. Ensuite, on peut même parler d'une attitude sarcastique de la part du locuteur ; l'introduction métalinguistique ("nach gärtnerischer Redeweise ») indique un ton ridiculisant et l'emploi du phraséolexème die Radieschen von unten ansehen confirme et renforce cette évaluation irrespectueuse, voire méprisante (fig. 2).

(13) Le terme construction prédicative figée (festgeprägte prädikative Konstruktion) est dû à Reichstein (1973).

(14) Pour plus de détails, $c f$. Lüger (2008: 201-202). 


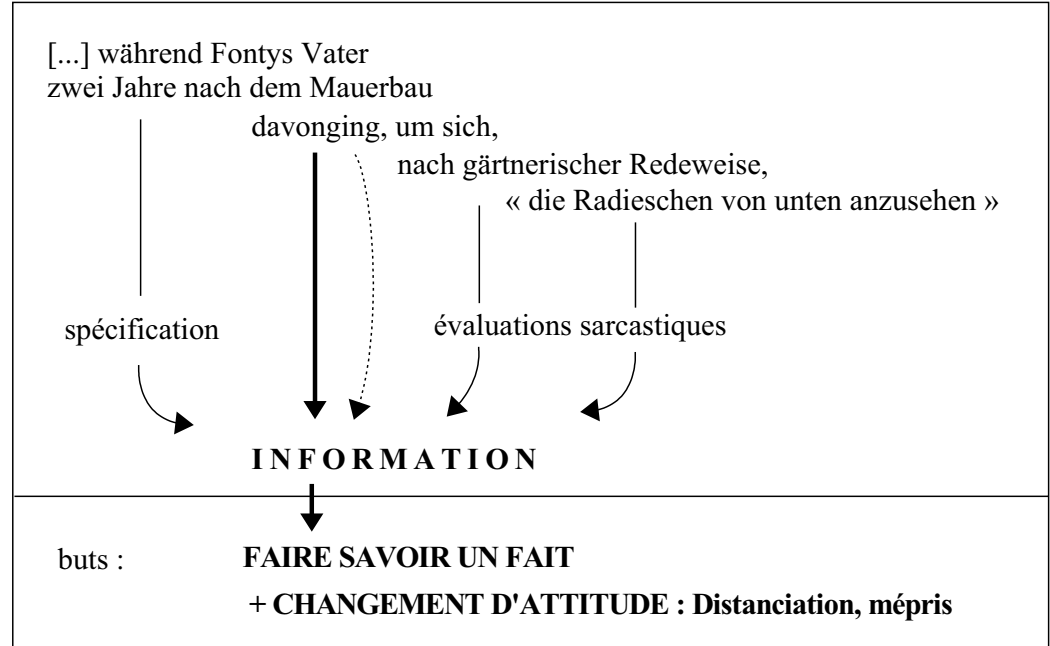

Fig. 2 : Expression figée et fonction textuelle

Quelles solutions ont été favorisées par les traducteurs? Surtout : Comment les évaluations sarcastiques ont-elles été reprises?

(18b) [...] tandis que c'est deux ans après la construction du Mur que le père de Fonty, selon son expression de jardinier, «alla voir les radis par en dessous» (235-236)

(18c) $[\ldots]$ mentre il padre di Fonty se ne andò " a vedere l'erba dalla parte delle radici», secondo il gergo dei giardinieri, due anni dopo la costruzione del Muro (207)

La version française omet d'abord une expression correspondante pour davonging et la structure finale ( $u m z u+$ infinitif) qui suit. En ce qui converne la locution die Radieschen von unten ansehen, on ne recourt pas au phraséolexème idiomatique manger les pissenlits par la racine, mais propose une traduction littérale qui ne rend donc pas le sarcasme du texte allemand. En (18c), on a affaire à une traduction plus fidèle : La locution vedere l'erba dalla parte delle radici correspond bien au texte de départ, et l'expression verbale andarsene reprend davongehen en (18a).

2.4. Jusqu'ici, il s'agit principalement d'un emploi d' expressions figées dont la traduction est marquée par une certaine réduction du sens. Mais il y a aussi des exemples où l'unité phraséologique et l'énoncé respectif sont purement et simplement supprimés. C'est le cas pour «Der nahm kein Blatt vor den Mund 》 déjà cité en (7) ; la phrase reste sans traduction dans le texte français (III, 50) ${ }^{(15)}$. Un exemple comparable :

(19) Der Unsterbliche hatte Dreck am Stecken $(\mathrm{V}, 90) \rightarrow \varnothing(85) \rightarrow$ L'Immortale aveva le mani sporche. (71)

Un problème presque insoluble résulte des multiples rapports textuels : Dans le texte on trouve à plusieurs reprises des expressions figées organisées en réseau et qui déploient des chaînes isotopiques. La traduction paraît d'autant plus difficile que ces rapports transgressent non seulement des phrases, mais aussi des paragraphes et parfois même des chapitres, comme les extraits en (20) l'illustrent bien :

(15) Dans le texte italien kein Blatt vor den Mund nehmen est traduit par la locution non aver peli sulla lingua (39). 
(20) (a)

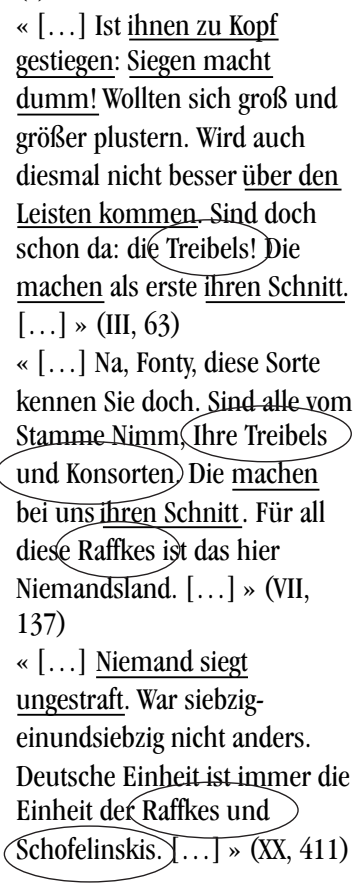

(b)

« [...] Ça leur est monté à la tête : la victoire rend idiot ! La grenouille qui veut se faire plus grosse que le bœuf. Ça ne tournera pas mieux cette foisci. Ils sont déjà là, les Treibel ! Ils seront les premiers à se tailler leur part. $[\ldots] »(\overline{61})$

\section{« [...] Et vous la connaissez} bien, cette espèce, Fonty. Tous de la tribu des "Je-prends", comme vos Treibel et consorts. Viennent chez nous se tailler leur part. Pour ces voraces, c'est une terre vierge, ici. $[\ldots] »(128)$ « [...] Il n'y a pas de victoire impunie. C'était la même chose en soixante-dix-soixante et onze. L'unité allemande c'est toujours l'unité des requins et des malfrats. [...]» (379-380) (c)

« [...] Si sono montati la testa: vincere fa diventare stupidi! Volevano gonfiarsi di più. Anche stavolta faranno il passo più lungo della gamba. Sono già arrivati i Treibel! Quelli per prima cosa pensano ai loro affari. [...]» $(48-49)$ « [...] Beh, Fonty, conosce bene questa risma. Sono tutti della razza Arraffa, i suoi Treibel e compagni. Vengono a mietere da noi. Per tutti questi pescecani qui c'è la terra di nessuno. [...]» (109) «[...] Nessuno vince impunemente. Non è stato diverso nel SettantaSettantuno. L'unità tedesca è sempre l'unità dei pescecani e degli spilorci. [...] » (334)

Ces passages sont des allusions au roman Frau Jenny Treibel oder ,, Wo sich Herz zum Herzen find't “ (1893) de Theodor Fontane. Il s'agit — dans les deux textes d'ailleurs - de mettre l'accent sur un comportement d'avidité ou de rapacité qui est attribué comme trait caractéristique aux « Treibel et consorts ». On peut constater que l'auteur se sert dans ce domaine d'un vocabulaire assez cohérent, et ceci à travers tous les chapitres du roman. La récurrence des désignations comme Raffkes et Schofelinskis est très typique ici, elle accentue ausssi bien les intentions matérialistes qu'une morale corrompue des protagonistes en question. Raffke s'emploie en général pour critiquer quelqu'un qui ne pense qu'à s'approprier de l'argent ou les biens des autres. Schofelinski n'est d'ailleurs pas une expression lexicalisée ; le mot est dérivé de schofel/schofelig " mesquin ».

Dans la version allemande, il y a, en outre, toute une série de moyens stylistiques, y compris des expression figées, qui confirment cette chaîne isotopique « rapacité / orgueil ». Quand on prend les extraits en (20b,c), on voit bien que ce phénomène n'a pas échappé aux traducteurs. Néanmoins, on peut se demander si la référence à une fable de La Fontaine (« La grenouille qui veut se faire aussi grosse que le bœuf ») convient dans ce contexte. De même, la locution se tailler la part fait penser à la part du lion, donc une association qui ne soutient pas non plus l'isotopie dominante. De plus, le lexème malfratévoque d'autres traits que Schofelinski, un terme qui est plutôt réservé à une qualification morale (et pas forcément criminelle). Dans (20c), on peut constater également que les rapports textuels sont moins marqués. Par exemple, la répétition de la locution seinen Schnitt machen n'a pas été retenue ; la traduction propose deux expressions différentes : pensare ai suoi affari et mietere da noi. Ensuite, l'énoncé « Wird auch diesmal nicht besser über den Leisten kommen » qui se présente en effet comme une constatation quasi- 
objective, est rendu à l'aide du phrasème fare il passo più lungo della gamba («se surmener ») ; ainsi, l'accent est mis davantage sur un défaut personnel. Pour la dénomination Raffke on aurait pu s'attendre — surtout à la suite de razza Arraffa au terme arrafatore, mais le traducteur a préféré pescecane (« requin »). Et finalement, le mot spilorcio ( « radin ») ne correspond que partiellement à Schofelinski — sans parler de l'originalité de cette création lexicale.

C'est donc, en fin de compte, la densité de la structure isotopique qui est en jeu. Mieux le traducteur réussit à trouver des unités phrasélogiques appropriées, à respecter le registre stylistique du vocabulaire et à relever la fonction de certaines récurrences, plus il sera possible de reconstruire les rapports textuels dans la langue cible.

\section{Conclusion}

Sans aucun doute, le roman de Günter Grass représente un texte de référence qui est à bien des égards exceptionnel : Tout d'abord il y la mise en scène de plusieurs époques et événements historiques, l'intégration d'un très grand nombre d'œuvres littéraires, et la plupart du temps les mentions explicites sont absentes; le lecteur doit donc essayer - et cela dans la mesure où son savoir historique, politique et littéraire le permet — de déchiffrer les allusions, de compléter ce qui est présenté en fragments et de construire un certain développement thématique. Le roman Ein weites Feld est aussi exceptionnel sous l'angle stylistique : En général, l'auteur ne se contente pas d'un emploi conventionnel des moyens syntaxiques et morphologiques, le domaine du vocabulaire et des expressions figées est particulièrement riche en modifications et en créations spéciales.

Dans le présent article il s'agissait surtout d'expliquer la polyfonctionnalité de quelques unités phraséologiques dans le roman et d'analyser de façon contrastive leur traduction en français et en italien. Malgré le nombre limité des occurences retenues certaines difficultés typiques et des cas d'intraduisibilité se sont dégagés.

En somme, entre l'équivalence totale et l'équivalence zéro on peut constater plusieurs gradations. Relativement peu problématiques sont les phrasèmes qui ne sont pas modifiés et pour lesquels il y a des formes analogues dans la langue cible. Les choses se compliquent pourtant quand on doit recourir à des quasi-synonymes ou à des paraphrases, toutes sortes de décalages sémantiques et pragmatiques peuvent donc s'imposer. D'autres questions se posent, quand on a affaire à des relations intertextuelles implicites, à un emploi ludique des expressions figées ou à un déploiement des chaînes isotopiques dépassant le cadre d'un chapitre. Ici, les limites de la traduisibilité sont vite atteintes.

Le but ne consistait pas à évaluer la qualité des deux traductions. Même si quelques exemples ont provoqué des remarques critiques, les traducteurs ont fourni un travail extraordinaire. Et cela d'autant plus que beaucoup de spécialistes pensent qu'on ne peut pas vraiment transposer les romans de Günter Grass dans une autre langue - un verdict qui a amené l'auteur à lancer aux traducteurs potentiels un appel à prendre plus de libertés et à se comporter comme des auteurs ${ }^{(16)}$.

(16) Grass : «Ich ermuntere die Übersetzer, sich den Text anzueignen, Beispiele aus ihrer eigenen Sprache einfließen zu lassen. Werdet Autoren! » (cité d'après 1e MDÜ - Fachzeitschrift für Dolmetscher und Übersetzer 57/3 (2011), 36). 


\section{Bibliographie}

BENGER, C.B. (2004), Von der 'Blechtrommel' bis 'Ein weites Feld': Günter Grass' Prosastil und die Probleme seiner Übertragung ins Französische. Freiburg (= Phil.Diss.).

Burger, H. $\left({ }^{1} 1998,{ }^{4}\right.$ 2010), Phraseologie. Eine Einführung am Beispiel des Deutschen. Berlin, Schmidt.

Burger, H., Buhofer, A., SiAlm, A. (1982), Handbuch der Phraseologie. Berlin, de Gruyter.

DOBROVOL'SKIJ, D. (2004), «Idiome und Übersetzung literarischer Texte », in : BrdarSzabó, R., Knipf-Komlósi, E. (eds) : Lexikalische Semantik, Phraseologie und Lexikographie. Festgabe für Regina Hessky. Frankfurt/M., Lang, 273-284.

EtTinger, St. (1994), "Phraseologische faux amis des Sprachenpaares Französisch-Deutsch », in : Sandig, B. (ed.) : Europhras 92. Tendenzen der Phraseologieforschung. Bochum, Brockmeyer, 109-136.

FLEISCHER, W. ( $\left.{ }^{1} 1982,{ }^{2} 1997\right)$, Phraseologie der deutschen Gegenwartssprache. Tübingen, Niemeyer.

ForgÁCS, E. (2004), «Phraseologismen als Mittel der Textkonstitution », in : PalmMeister, Ch. (ed.) : Europhras 2000. Tübingen, Stauffenburg, 123-135.

GONZÁLEZ REY, I. (2002), La phraséologie du français. Toulouse, Presses Universitaires du Mirail.

GRÉCIANO, G. (1983), Signification et dénotation en allemand. La sémantique des expressions idiomatiques. Paris, Klicksieck.

KLimaszewsKa, Z. (1992), « De fraseologie van het Nederlands, Duits en Pools vanuit een cultuurhistorisch gezichtspunt », in : Hipp, H. (ed.) : Niederlandistik und Germanistik. Festschrift für Gerhard Worgt. Frankfurt/M., Lang, 129-137.

KOLLER, W. (1974), « Intra- und interlinguale Aspekte idiomatischer Redensarten », Skandinavistik 4, 1-24.

— $\left({ }^{4}\right.$ 1992), Einführung in die Übersetzungswissenschaft. Heidelberg, Quelle u. Meyer.

— (1994), «Phraseologismen als Übersetzungsproblem», in : Sandig, B. (ed.) : Europhras 92. Tendenzen der Phraseologieforschung. Bochum, Brockmeyer, 351-373.

LÜGER, H.H. (1999), Satzwertige Phraseologismen. Eine pragmalinguistische Untersuchung. Wien, Edition Praesens.

— (2004), "Kollokationen - zwischen Arbitrarität und Kompositionalität. », in : Pohl, I., Konerding, K.P. (eds) : Stabilität und Flexibilität in der Semantik. Frankfurt/M., Lang, 45-66.

— (2008), «Mehrwortverbindungen in der Übersetzung - ein weites Feld », in : Baudot, D., Kauffer, M. (eds) : Wort und Text. Festschrift für René Métrich zum 60. Geburtstag. Tübingen, Stauffenburg, 195-203.

Mohr-ElfadL, S. (1999), «Jeu de Tambour: La phraséologie dans l'œuvre de G. Grass et sa traduction », Nouveaux Cahiers d'Allemand 17, 401-411.

PERRIN, L. (2011), "Figement, énonciation et lexicalisation citative », in: Anscombre, J.-C., Mejri, S. (eds) : Le figement linguistique : la parole entravée. Paris, Champion, 81-94.

REICHSTEIN, A.D. (1973), «Zur Analyse der festgeprägten Sätze im Deutschen », Deutsch als Fremdsprache 10, 212-222.

RÖHRICH, L. ( $\left.{ }^{3} 1982\right)$, Lexikon der sprichwörtlichen Redensarten, 4 t. Freiburg, Herder. 
SCHAPIRA, Ch. (1999), Les stéréotypes en français. Proverbes et autres formules. Paris, Ophrys.

SCHMALE, G. (2011), « Was ist in der Sprache „vorgeformt“? Überlegungen zu einer erweiterten Definition sprachlicher Präformiertheit », in : Schäfer, P., Schowalter, Ch. (eds) : In mediam linguam. Mediensprache - Redewendungen Sprachvermittlung. Landau, VEP, 177-190.

SCHWEIZER, B.M. (1978), Sprachspiel mit Idiomen. Eine Untersuchung am Prosawerk von Günter Grass. Zürich, Juris.

SteIN, St. (2001), «Formelhafte Texte. Musterhaftigkeit an der Schnittstelle zwischen Phraseologie und Textlinguistik », in : Lorenz-Bourjot, M., Lüger, H.H. (eds): Phraseologie und Phraseodidaktik. Wien, Edition Praesens, 21-39.

\section{questions de communication 23-2013}

Figures du sacré

Extension du domaine du sacré

S. Dufour, J.-J. Boutaud

L'Incarnation comme communication, ou l'auto-communication de Dieu en régime chrétien

\section{$D$. Douyère}

Le pélerinage aujourd'hui : entre solidarité et hagiothérapie

\section{A. Bernard}

Le réligieux et le sacré vus à travers l'iconographie de l'olfaction

M. Giulia Dondero

Université : la hargne, la rogne et la grogne. Mais encore ?

\section{B. Fleury, J. Walter}

Les classements des établissements d'enseignement supérieur et de recherche : des miroirs déformants aux instruments de régulation

\section{J. Bouchard}

Pas de démocratie sans courage des universitaires

É. Dacheux
Les représentations du sacré chez les élèves-officiers de l'armée de l'air. Entre mutations et permanence de la notion d'engagement

C. Batazzi, R. Delaye

Un anthropologue à l'Apple Store.

Notes de terrain sur le millénarisme d'Apple

\section{P. Lardellier}

(Non-)Témoignage. L'expérience du sacré : la parole et le geste

\section{A. Cognard}

Retour sur l'évolution universitaire en France

J. Duval

La dérive des universités, vue de l'autre côté de l'océan Atlantique É. George «Dérapage » ou remise en cause de l'institution universitaire

G. Labelle, É. Martin, M. Ouellet Les universités belges entre concurrence et excellence

M. Lits, É. Léonard

PRIX AU NUMÉRO 20 euros (frais de port de 3,25 euros en sus)

ABONNEMENT ( 1 an, 2 numéros) 32 euros (frais de port de 6,50 euros en sus) Abonnement: Presses universitaires de Nancy•pun@univ-nancy2.fr Achat en ligne : http://www.lcdpu.fr/collections/questionsdecommunication En ligne: questionsdecommunication.revues.org 